\title{
On the Foundation of Space and Time by Quantum-Events
}

\author{
Andreas Schlatter ${ }^{1}$ (1)
}

Received: 12 May 2021 / Accepted: 18 November 2021 / Published online: 1 December 2021

(c) The Author(s) 2021

\begin{abstract}
The true nature of space and time has been a topic of natural philosophy, passed down since the presocratic era. In modern times reflection has particularly been inspired by the physical theories of Newton and Einstein and, more recently, by the quest for a theory of quantum gravity. In this paper we want to specify the idea that material systems and their spatio-temporal distances emerge from quantum-events. We will show a mechanism, by which quantum-events induce a metric field between material systems, which is governed by Einstein's equation including a cosmological constant.
\end{abstract}

Keywords Quantum-event · Space-time · Emerging gravity · Transactional interpretation $\cdot$ Causal sets $\cdot$ Einstein equation $\cdot$ Cosmological constant

\section{Introduction}

There has been philosophical reflection about the nature of space and time, passed down to us since presocratic times. In the modern era this reflection has been part of natural philosophy, which aims to describe nature by the language of mathematics. Over time two main traditions have emerged. On the one side there is the position prominently taken by Newton, called the substantival position, which claims that space and time are absolute entities of their own, a theatre, within which matter resides and interacts [1]. On the other side there is the relational tradition represented by Leibniz, which believes that space and time are emerging metric relations between material systems [1]. It is probably fair to say that all the developments since the two great thinkers formulated their views can be attributed (more or less) to one or the other tradition. The philosopher Mach, who influenced the young Albert Einstein very much, clearly belonged to the second camp, whereas the standard ontology of general relativity today, ironically, is closer to the first one, by attributing to the metric field physical properties like energy with notable difference to

Andreas Schlatter

schlatter.a@bluewin.ch

$1 \quad$ Küttigen, Switzerland 
Newton insofar that it is dynamical itself and on eye-level with matter, in contrast to being just a background stage [1].

In the context of this debate belongs, since the time of philosophers like Parmenides and Heraklit [2], the question whether in nature there is true, irreversible change or whether change and the flow of time are just an illusion. Today's blockworld ontology in physics is in point of fact in the camp of Parmenides, denying that time is fundamentally real. Opposed to this is the stubborn human experience that there is directed change and past and future are very distinct existential regions in time, irreversibly following each other.

In this work we want to base on the idea that quantum-events, yet to be defined precisely in paragraph 3, are real physical facts, fundamental to change and causing the empiric realm of localized material systems and their spatio-temporal distances to emerge. We will call this realm "empirical space-time" throughout the paper. We will see that space and time, though being of different nature, form a union and that a metric relation, governed by Einstein's equation with a cosmological term, naturally emerges from the event-mechanism. The description of quantum-events [3-6] follows ideas from the transactional and causal-set interpretation of quantum mechanics. We thus attempt to establish a link between gravity and quantum mechanics relying entirely on the existing formalism, but making a distinct assumption on the reality of quantum-events and their consequences. By the way of preparation, we first give a motivation, ${ }^{1}$ why the formalism of quantum mechanics is suited to describe nature so aptly. To do this, we start with some very general thoughts.

\section{Properties and Change}

In the fundamental debate about space and time, as in any fundamentally philosophical discussion, one has to start with some basic, irreducible notions, from where a theory is built. In our case, we take the notion of physical "property" of a system as the starting point. A property of a set $\Sigma$ of physical systems is a set $\mathcal{P}$ of values, which a physical system $\sigma \epsilon \Sigma$ can assume $\pi_{\sigma} \epsilon \mathcal{P}$. ${ }^{2}$ There are, of course, several different kinds of properties, which a system can have, and we speak of a "unique identifying property", if there holds for $\sigma, \hat{\sigma} \epsilon \Sigma$ and $\underline{\pi}_{\sigma}, \underline{\pi}_{\hat{\sigma}} \epsilon \mathcal{P}$

$$
\underline{\pi}_{\sigma}=\underline{\pi}_{\hat{\sigma}} \Longleftrightarrow \sigma=\hat{\sigma} \text {. }
$$

Unique identifying properties as well as physical systems are represented by suitable models, which explain the data, but often also serve as ontic representations. Although this identification can be ontologically difficult, especially if one believes in "the thing in itself", it is normally all we have and is at least sufficient for doing physics. Empiricism would even say it is all we can have.

\footnotetext{
1 There are attempts to rigorously derive the formalism of QM from first principles. This is not our aim, though.

2 The bar-sign indicates that there might be several components.
} 
There are two other notions, which in connection with properties become central: they are "change" and "identity". It is an empirical fact that a physical system $\sigma \epsilon \Sigma$ can assume distinct values of a property, while still being identified as the same system. But identity-preserving difference of values of a unique identifying property leads to a contradiction to condition (1), unless we introduce a dependency from (at least) one additional parameter $\tau \epsilon \Theta$, satisfying for $\sigma_{i}, \sigma_{j} \epsilon \Sigma \underline{\pi}_{\sigma_{i}}, \underline{\pi}_{\hat{\sigma}} \epsilon \mathcal{P}$

$$
\left(\underline{\pi}_{\sigma_{i}}\left(\tau_{i}\right) \neq \underline{\pi}_{\sigma_{j}}\left(\tau_{j}\right)\right) \wedge\left(\sigma_{i}=\sigma_{j}\right) \Rightarrow\left(\tau_{i} \neq \tau_{j}\right) .
$$

The parameter $\tau \epsilon \Theta$ is hence a logical parameter, which we call "time". A difference of values of a particular system at different times $\tau \epsilon \Theta$ is, what we call "change". Were it not for this identity preservation, we would speak of "creation" instead and a physical system would hardly have a "history". Clearly, there might hold $\tau=\tau(\sigma, \underline{\pi})$ and because of our experience of "before" and "after", the set $\Theta$ should allow an (at least partial) order relation. Given a system $\underline{\pi}_{0}=\underline{\pi}\left(\tau_{0}\right) \epsilon \mathcal{P}^{3}$ we can define an "evolution" to be a map $U: \Theta \rightarrow \mathcal{P}, \tau \mapsto U\left(\tau, \underline{\pi}_{0}\right), U\left(\tau_{0}, \cdot\right)=\mathbb{Q}_{\mathcal{P}}$. We call the set $\left\{U\left(\tau, \underline{\pi}_{0}\right), \tau \epsilon \Theta\right\} \subset \mathcal{P}$ the path of $\underline{\pi}_{0}$. Because of (2) we have for all $\tau \epsilon \Theta$ and different $\underline{\pi}_{0}, \underline{\pi}_{0} \epsilon \mathcal{P}$

$$
U\left(\tau, \underline{\pi}_{0}\right) \neq U\left(\tau, \underline{\tilde{\pi}}_{0}\right)
$$

Hence the "paths" of different systems never intersect and for every $\tau \epsilon \Theta$ the map $U(\tau, \cdot): \mathcal{P} \rightarrow \mathcal{P}$ is a bijection. Note that at this point and without further structure, there is no notion of dynamics and the path is just an ordered set of values $\underline{\pi}(\tau) \epsilon \mathcal{P}$, representing the physical system as a whole.

This paper will concentrate on space, which we introduce as a property of matter in the sense that a material system can have a position in space, and the notion of time induced by change of position. We will see that position in space-time is a unique identifying property. To get there and beyond, we now have to consider concrete models of nature in terms of our framework, namely specific triples $(\mathcal{P}, \Theta, U)$.

\section{Models of Nature}

To illustrate the abstract framework, introduced in paragraph 1, we consider briefly classical physics and then, in more detail, quantum-physics.

\subsection{Classical Physics}

The basic classical physical systems are massive point-particles and fields in space and time. The models are $\left(\mathbb{R}^{3}, \mathbb{R}, \Phi_{t}\right)$ and $\left(C^{1}\left(\mathbb{R}^{3}, \mathbb{R}^{3}\right), \mathbb{R}, \Phi_{t}\right)$, respectively, where $\Phi_{t}: \mathbb{R} \rightarrow \mathbb{R}^{3}\left(C^{1}\left(\mathbb{R}^{3}, \mathbb{R}^{3}\right)\right)$ are flows solving suitable differential equations to given

\footnotetext{
3 Note that we identify the system with its unique identifying property.
} 
initial conditions. ${ }^{4}$ Three-dimensional space is a unique identifying property, since no two particles can at the same time be at the same point in space, nor can two different fields coincide in all space points at a given time. $\mathcal{P} \times \Theta \stackrel{\text { def }}{=} \mathbb{R}^{3} \times \mathbb{R}$ is suitable, since the real line is (totally) ordered and the continuum allows calculus. There is considerable doubt, however, whether time and space really are continuous and $\mathbb{R}^{3} \times \mathbb{R}$ is the right model. While the triples $\left(\mathbb{R}^{3}, \mathbb{R}, \Phi_{t}\right),\left(C^{1}\left(\mathbb{R}^{3}, \mathbb{R}^{3}\right), \mathbb{R}, \Phi_{t}\right)$ base on a unique identifying property, they are adynamical in the sense that $\Phi_{t} \subset \mathbb{R}^{3}\left(C^{1}\left(\mathbb{R}^{3}, \mathbb{R}^{3}\right)\right)$ exists as a subset and there is no dynamics and no direction, since the equations leading to the flow are time-reversible. We encounter ultimately a static picture, which is further strengthened by the fact that often $\Phi_{t}$ results from a variational principle with functionals defined over all space and time. The ultimate choice of $(\mathcal{P}, \Theta, U)$ in quantum physics achieves more, as we will now show. It first combines space and time to space-time as a unique identifying property for material systems and secondly provides a mechanism for the notion of becoming and time-direction.

\subsection{Quantum Physics}

The big step in quantum physics is the idea that the properties of physical systems are not measured values themselves but amplitudes, which combine to probabilities for individual values. For a single system we have technically in the non-relativistic case $\mathcal{P} \stackrel{\text { def }}{=}\left\{\underline{c}_{\underline{x}} \epsilon L_{\mathbb{C}}^{2}\left(\mathbb{R}^{3}\right),\|\underline{c}\|_{2}=1\right\}=S_{\mathbb{C}}^{2}\left(\mathbb{R}^{3}\right)$. We interpret the expression $\underline{c}_{\underline{x}}^{*} \underline{\underline{c}} \underline{\underline{x}}=\left.|\underline{\underline{c}}|_{\underline{x}}\right|^{2}$ as the probability density for a system to be at point $\underline{x} \in \mathbb{R}^{3}$. As in classical physics, we set $\Theta \stackrel{\text { def }}{=} \mathbb{R}$ and the evolution will just be maps $U_{t}: \mathbb{R} \rightarrow S_{\mathbb{C}}^{2}\left(\mathbb{R}^{3}\right)$ belonging to the unitary group $U\left(L_{\mathbb{C}}^{2}\left(\mathbb{R}^{3}\right)\right)$. So we have the triple $\left(S_{\mathbb{C}}^{2}\left(\mathbb{R}^{3}\right), \mathbb{R}, U_{t}\right)$. Lie-group theory tells us that $\underline{c}_{\underline{x}}(t)=U_{t}\left(\underline{c}_{0 \underline{x}}\right)=e^{-\frac{i}{\hbar} H t} \underline{c}_{0 \underline{x}}$ for some Hermitian $\operatorname{HeL}\left(L_{\mathbb{C}}^{2}\right)$, and hence provides a differential equation for evolution $i \hbar \frac{d}{d t} \underline{c}_{\underline{x}}(t)=\frac{d}{d t} U_{t}\left(\underline{c}_{0 \underline{x}}\right)=H \underline{c}_{\underline{x}}(t)$. So far, one thinks of $\mathbb{R}^{3} \times \mathbb{R}$ as a topological space, endowed with the standard metric.

Next to material systems in the narrow sense, there are electromagnetic fields with their quanta, the photons. Photons have a key property, namely that their energy and three-momentum, $\vec{p}$, always satisfy

$$
\frac{E}{|\vec{p}|}=c .
$$

Here the constant $c$ denotes the speed of light. Using Einstein's and de Broglie's relation, $E=h v=\frac{h}{\Delta t}$ and $|\vec{p}|=\frac{h}{\lambda}=\frac{h}{\Delta x}$, respectively, Eq. (4) can be formulated in space and time by

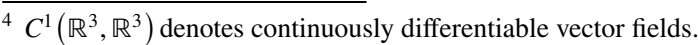




$$
\frac{E}{|\vec{p}|}=\frac{\Delta x}{\Delta t}=c .
$$

Since Eq. (5) must hold for every photon, Galilean-type transformations of space and time $\mathbb{R}^{3} \times \mathbb{R}$ should not change it and we are lead to the Lorentz (Poincaré) group $\Lambda$ of appropriate symmetry transformations, which unite space and time to Minkowski space-time $\mathbb{M}^{4}$. The symmetry group $\Lambda$ has different representations $D_{\Lambda}^{s}$, corresponding to vector spaces $V^{D_{\Lambda}^{s}}$ and classified by the spin-number $s=\frac{k}{2}, \mathrm{k} \in \mathbb{N}_{0}$. Since the evolution has to respect the symmetries, the triple $(\mathcal{P}, \Theta, U)$ becomes $\left(S_{\mathbb{C}}^{2}\left(\mathbb{M}^{4}, V^{D_{\Lambda}^{s}}\right), \mathbb{R}, U_{t}^{D_{\Lambda}^{s}}\right)$, where $U_{t}^{D_{\Lambda}^{s}} \epsilon U\left(V^{D_{\Lambda}^{s}}\right)$ is an element of the unitary group over $V^{D_{\Lambda}^{s}}$. Matter in the narrow sense has spin number $s=\frac{1}{2}$ and there holds by the rules of spin-statistics that the space for multiple, independent systems is the anti-symmetric product $\bigwedge S_{\mathbb{C}}^{2}\left(\mathbb{M}^{4}, V^{D_{\Lambda}^{s}}\right)$. This means that the property $\underline{c}_{\underline{x}}^{s}, s=\frac{1}{2}$, is unique identifying in our sense, since the components of a system consisting of several independent parts must all have different amplitudes. In a final step of abstraction, so called second quantization, the amplitudes become operators $\underline{\underline{c}}_{\underline{x}}^{s}, \widehat{\underline{c}}_{\underline{x}}^{s \dagger} \in \mathcal{D}^{s}(L(\mathcal{F}))^{5}$ acting on multi-particle state spaces $\mathcal{F}$. These operators are also called quantumfields, have a corresponding evolution map $U_{t}^{\mathcal{D}^{s}}$ and are interpreted to create and annihilate particles at some space-time location $x \in \mathbb{M}^{4}$. Our findings so far, are valid for the triple $\left(\mathcal{D}^{s}(L(\mathcal{F})), \mathbb{R}, U_{t}^{\mathcal{D}^{s}}\right)$ too, but we will continue to argue with amplitudes in $S_{\mathbb{C}^{*}}^{2}{ }^{6}$ The field-view is important, however, for a relativistic mechanism of quantum-events, as described in paragraph 3.

The evolutions $U_{t}$ in quantum physics suffer from the same time-symmetry and adynamism as the ones in the classical case do. Unitary-only quantum mechanics suffers in addition from paradoxes like Schrödinger's cat or Wigner's friend, which until today cause controversies [7]. But the choice of $\mathcal{P}$ offers a way out. The evolution $U_{t}\left(\underline{c}_{0 \underline{x}}\right)$ may spontaneously be broken and collapse, $U_{t}\left(\underline{c}_{0 \underline{x}}\right) \rightarrow \underline{\delta}_{\underline{x}_{0}}$, to concentrate around some $\underline{x}_{0} \in \mathbb{M}^{4}$. We are going to describe this mechanism by way of giving a concise account of the transactional interpretation of quantum mechanics, to which collapse is a central piece and according to which the flat spatio-temporal structure, $\mathbb{M}^{4}$, emerges from quantum-events.

\footnotetext{
${ }^{5}$ The correct mathematical notion is operator-valued distributions.

${ }^{6}$ An amplitude for quantum-fields would look like $\left\langle 0\left|\left\{T\left(\widehat{\widehat{c}}_{\underline{y}} \widehat{\underline{c}}_{\underline{x}}{ }^{\dagger}\right)\right\}\right| 0\right\rangle$, where $T$ is the time-order operator and $|0\rangle$ the vacuum state. It denotes the amplitude to create a particle at $\underline{x \in \mathbb{M}^{4}}$ and to annihilate one at $\underline{y \in} \mathbb{M}^{4}$.
} 


\section{Quantum-Events and Light-Clocks}

\subsection{Quantum-Events}

Quantum amplitudes of closed, isolated systems are represented as unit vectors $\underline{c}_{\underline{x}}^{s} \in S_{\mathbb{C}}^{2}\left(\mathbb{M}^{4}, V^{D_{\Lambda}^{s}}\right)$, also called quantum states. In the transactional interpretation [8] a quantum state $\underline{c}_{\underline{x}}^{s}$ is launched as an "offer-wave" by an emitter and gets possible responses by "confirmation-waves", represented by dual vectors $\underline{c}_{\underline{y}}^{s *}$ launched by possible localized absorbers. This process is called an incipient transaction. The selection of a specific "response" $\underline{c}_{\underline{y}}^{s *}$ is purely random and leads to a "transaction", which is the actualization of absorption and emission as real events in space-time, and whose probability (density) is $\left|\delta_{\underline{y}} * c_{\underline{x}}^{s}\right|^{2}=\left.|\underline{\underline{c}} \underline{\underline{y}}|^{s}\right|^{2}$ The relativistic transactional interpretation [3] offers additionally an explanation, why offer-waves (and confirmation-waves) are actually being created. Relativistic interactions can be thought of as the mutual exchange of virtual bosons by quantum fields, creating possibilities in a pre-space-time process. Transactions, in turn, are triggered by the exchange of real bosons and their four-momentum between emitter and absorber. The general amplitude for emission and absorption of real bosons is the coupling amplitude between matter-and gauge fields and a specific transaction can happen, if the conservation laws are satisfied. By the exchange of four-momentum the quantum states of emitter and absorber collapse and the physical systems are said to be at the corresponding space-time points (regions). We will use the term "event-radiation" for the momentum transfer in transactions and mean the entire transaction-mechanism, if we speak of quantum-events. While in [8] an incipient transaction is thought to be a process within space-time, it is a pre-space-time process and constituent for empirical space-time in [3] in order to overcome certain challenges [9].

Space-time thus becomes the connected set of emission-and absorption points, between which space-time intervals are being created through the four- momentum of the event radiation. It is here, where the transactional view touches causal-set theory $[4,5]$, in which events spread in space-time by a stochastic Poisson-process. A transaction by boson exchange, understood as a decay-process in quantum field theory and hence Poissonian [10], is then a special case in this model. Successive emission and absorption events constitute a causal chain. On the other hand, the causal-set approach creates volume around single events in the manifold, whereas the transactional approach is relational and creates space-time intervals between two events. Note, that the actualization of a space-time interval amounts to spontaneously breaking the unitary evolution $U_{t}$ of the quantum states. At the same time the choice of exchanged four-momentum selects a space-direction, whereas

\footnotetext{
7 Note, that we are idealizing, since, strictly speaking, individual points $\underline{x} \in \mathbb{M}^{4}$ are null-sets and no probability can be assigned.
} 
a time-direction is a priori determined by the choice of the absorber phase, which selects the propagation of positive (or negative) energy from emitter to absorber. ${ }^{8}$ Because there is no preferred emission-direction, space is isotropic, and because the whole mechanism is indifferent to specific space-time points, space-time is homogeneous. We will in the sequel focus on the electromagnetic interaction and the related exchange of photons.

By what we have explained so far, we have motivated the idea that the formalism of quantum physics is suited to explain the emergence of empirical flat space-time by the mechanism of quantum-events. Event radiation creates metric relations between localized emitters and absorbers and the mechanism contains therefore an intrinsic way to measure time-intervals by means of the exchanged photons. We will now show that this event-mechanism also generates the gravitational field i.e. a curved metric of empirical space-time, governed by Einstein's equation.

\subsection{Light-Clocks}

It takes a general (closed and isolated) quantum-system, represented by a vector in some Hilbert space, $\psi_{0} \epsilon H_{\mathbb{C}}$, with average energy above ground state $\left(\bar{E}-E_{0}\right)$, minimally a time interval of

$$
\overline{\Delta t}=\frac{h}{4\left(\bar{E}-E_{0}\right)}
$$

in order to evolve into an orthogonal state $\psi_{1} \epsilon H_{\mathbb{C}},\left\langle\psi_{0} \mid \psi_{1}\right\rangle=0$ [11]. We can use such a system as the core of a clock, i.e. an abstract periodic process, without ability to "indicate" time [12], and after period $\overline{\Delta t}$ we can be sure that a period has passed [13]. In our case, we will chose photons with energy $\left(E-E_{0}\right)=h v$, to get (after normalization by a factor four) corresponding light-clocks with period

$$
\overline{\Delta t}=\frac{1}{v}
$$

A light clock is best imagined classically as a light signal, which passes a wavelength $\lambda=\frac{c}{v}$ in a time interval $\Delta t=\frac{1}{v}$ in an inertial frame. ${ }^{9}$ We will also encounter situations, where there is not a single oscillator, but many of them over a range of frequencies in thermal equilibrium, and where the energy is given by a temperature $T$. For oscillators with $\bar{E}_{v} \approx k_{B} T$ (i.e. $h v \ll k_{B} T$ ), we get a corresponding light clock

$$
\overline{\Delta t}=\frac{h}{k_{B} T} .
$$

\footnotetext{
${ }^{8}$ This choice amounts to the selection of either the Feynman or the Dyson propagator. The standard approach is to take the Feynman propagator.

${ }^{9}$ Of course, the invariant four-distance, which the photon "passes" is zero.
} 
We call the special light-clock in (8) a thermal clock. We are now in a position to start the main endeavor of this paper, namely to show how quantum-events and the corresponding event-radiation lead to a metric relation on emerging space-time, governed by Einstein's equation.

\section{The Structure of Space-Time}

\subsection{Synchronizing Light-Clocks}

The concept of a thermal clock (8) unfolds its power, if we consider multiple events of interacting quantum-systems. Multiple events manifest themselves in space-time by acceleration. In $\mathbb{M}^{4}$ physical systems of constant acceleration $\kappa$ in $x$-direction, say, can be expressed in Rindler-coordinates. This happens by choosing a co-moving coordinate system, defined in the wedge limited by $|x|=t$, and given by the transformations

$$
x=\rho \cosh (\kappa \vartheta), t=\rho \sinh (\kappa \vartheta), \varrho \geq 0,-\infty<\vartheta<\infty .
$$

The corresponding line-element is

$$
d s^{2}=\left(\frac{\kappa \varrho}{c^{2}}\right)^{2} c^{2} d \vartheta^{2}-d \varrho^{2}-d y^{2}-d z^{2} .
$$

Contrary to velocity, acceleration is not purely perspectival and cannot be transformed away by a Lorentz transformation. ${ }^{10}$ But there are local inertial reference-frames at $t=\vartheta=0$, where systems are instantaneously at rest. By the Tolman-Ehrenfest effect [15] we have in thermal equilibrium for systems being instantaneously at rest and located at arbitrary $\varrho_{1}, \varrho_{2}$

$$
T_{\varrho_{1}} \frac{\kappa \varrho_{1}}{c} d \vartheta=T_{\varrho_{2}} \frac{\kappa \varrho_{2}}{c} d \vartheta
$$

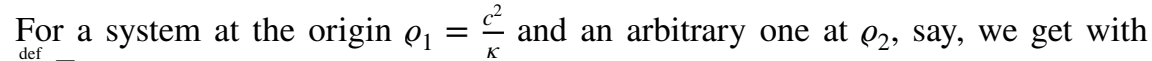
$T_{\kappa} \stackrel{\text { def }}{=} T_{\varrho_{1}}$

$$
T_{\kappa} \frac{c^{2}}{\kappa}=T_{\varrho_{2}} \varrho_{2}=\text { const. }
$$

The constant on the right does no longer depend on $\kappa$. Assume that in this chart (coordinate system) there is a thermal bath of temperature $T_{\kappa}$, and we want to gauge proper time by a corresponding thermal clock. By (6), (8) and (12) we get for a system instantaneously at rest at the origin and with $d \widehat{s}=\frac{c}{\kappa} d s$

\footnotetext{
$\overline{10}$ Acceleration is defined by $a=\frac{\Delta v}{\Delta t}=\frac{\Delta}{\Delta t}\left(\frac{\Delta x}{\Delta t}\right)=\frac{\left(x_{2}-x_{1}\right)-\left(x_{1}-x_{0}\right)}{\left(t_{2}-t_{1}\right)\left(t_{1}-t_{0}\right)}$ and involves at least two transactions. It therefore differs from inertial motion in an absolute way [14].
} 


$$
d \tau=\frac{d \widehat{s}}{\overline{\Delta t}}=\frac{4}{h} k_{B} T_{\kappa} \frac{c^{2}}{\kappa} d \vartheta
$$

We want to fix the constant in (12) and for this purpose synchronize ${ }^{11}$ (13) with a quantum-clock, defined by a matter-wave with rest mass $m_{0}$, frequency $\omega=2 \pi v$ and corresponding acceleration $\kappa_{\omega}$. In its respective oscillatory rest-frame and for $m_{0} \ll \frac{\hbar \omega}{c^{2}}$, the matter-clock measures time in analogy to (13) in units of

$$
d \tau_{\omega}=\frac{4}{h} E_{\omega} \frac{c^{2}}{\kappa_{\omega}} d \vartheta
$$

By the de Broglie-relation there holds with $k=|\vec{k}|$ denoting the wave number

$$
E_{\omega}^{2}=\hbar^{2} \omega^{2}=c^{2} \hbar^{2} k^{2}+m_{0}^{2} c^{4} .
$$

Further with $u_{\omega}=\frac{\omega}{k}$ and $v_{\omega}=c^{2} \frac{k}{\omega}$ denoting the phase-and group velocity, respectively, we have

$$
\kappa_{\omega}=2 \pi u_{\omega} \omega .
$$

By (15) and (16) Eq. (14) turns into

$$
d \tau_{\omega}=\frac{4}{h} \hbar \omega \frac{c^{2} k}{2 \pi \omega^{2}} d \vartheta=\frac{c^{2} k}{\pi^{2} \omega} d \vartheta=\frac{v_{\omega}}{\pi^{2}} d \vartheta .
$$

If we synchronize the two clocks, $d \tau=d \tau_{\omega}$, we therefore get

$$
\frac{4}{h} k_{B} T_{\kappa} \frac{c^{2}}{\kappa}=\frac{v_{\omega}}{\pi^{2}} .
$$

For the temperature $T_{\kappa}$ this implies

$$
T_{k}=\frac{\hbar \kappa v_{\omega}}{2 \pi k_{B} c^{2}} .
$$

Expression (19) is a generalized Davies-Unruh temperature. If we choose a massless wave $\left(m_{0}=0\right)$, then we are in the situation $u_{\omega}=v_{\omega}=c$ and (19) turns into the familiar Davies-Unruh temperature formula $[16,17]$

$$
T_{\kappa}=\frac{\hbar \kappa}{2 \pi k_{B} c} \text {. }
$$

We will use formula (20) in the next paragraph in a concrete physical situation.

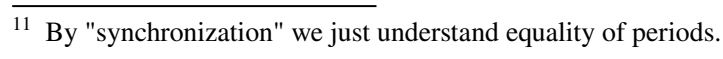




\subsection{Gravitational Acceleration}

We now want to generalize our approach by assuming that space-time is just locally flat. ${ }^{12}$ In order to apply formula (20) we must have an appropriate acceleration. Of course, we want it to be gravitational acceleration and we will show, how transactions can indeed give rise to local gravitational acceleration $g_{R}$.

The following argument bases on an exposition in [18]. Let a quantum-event be given by two physical systems of mass $m$ and $M$, respectively, which come into being by a photon-transaction in locally flat space-time at relative rest and distance $R$ to each other. Let further an elementary bit of information be connected to the existence or non-existence of a physical system in space-time. Since a photon offerwave is a priori emitted symmetrically in all space-directions, we find the photon at time $\Delta t=\frac{1}{v}=\frac{R}{c}$ on the surface of the sphere with radius $R$ around $M$. The sphere can be considered as a storage device for position-information whose total amount is proportionate to the surface area $A_{R}$. By introducing a constant $\widehat{G}$, which will be identified with the gravitational constant below, we can write for the number of bits $N_{R}=\frac{A_{R} c^{3}}{\widehat{G} \hbar} .{ }^{13}$ We may also assume that a bit of information is part of the surfaceinformation, once it is at a distance of its Compton length $\lambda=\frac{\hbar}{m c}$ from the sphere, and that this information changes linearly with the distance $0 \leq \Delta x \leq \frac{\hbar}{m c}[18,19] .{ }^{14}$ This holds because structureless systems can reasonably be supposed to have the size of their Compton-length. So a quantum-event causes an entropy change of

$$
\Delta S=2 \pi k_{B} \frac{m c}{\hbar} \Delta x .
$$

For the total energy $E$ within the ball of radius $R$ we have by the holographic principle $^{15}$ and under assumption of even distribution over the bits

$$
E=M c^{2}=\frac{1}{2} k_{B} N_{R} T
$$

The number $T$ is the surface-temperature on the sphere of radius $R$. By introducing the Planck-length $l_{P}=\sqrt{\frac{G \hbar}{c^{3}}}$ (here $G$ is Newton's constant) we indeed get for the number of bits if $G \equiv \widehat{G}$

$$
N_{R}=\frac{A_{R}}{l_{P}^{2}}=\frac{A_{R} c^{3}}{\widehat{G} \hbar}=\frac{4 \pi R^{2} c^{3}}{G \hbar} .
$$

By (22) and (23) we derive for the surface-temperature $T$

\footnotetext{
12 "Locally flat" means approximately flat in small regions around a point $x_{0}$. Technically this amounts to $g_{a b}\left(x_{0}\right)=\eta_{a b}, \Gamma_{i j}^{k}\left(x_{0}\right)=0$, but generally $\Gamma_{i j, v}^{k}\left(x_{0}\right) \neq 0,0 \leq i, j, k, v \leq 3$.

13 The constant $\widehat{G}$ could actually be defined by $\widehat{G}=\frac{A_{R} c^{3}}{N_{R} \hbar}$.

14 This assumption implies $R>\lambda$.

15 The holographic principle is a generalization of a result found for black-hole entropy [19] and says hat the total amount of information within a region of space is found on its surface, see e.g. [20].
} 


$$
T=\frac{M G \hbar}{2 \pi R^{2} k_{B} c} .
$$

Entropy in (21) increases with the distance $\Delta x$ from the surface and hence an attractive force $F$ acts as the result of a kind of "osmotic pressure" from the region of higher entropy outside the sphere towards the one of lower entropy inside. For the total energy-change on the surface we have the entropic-force equation

$$
\Delta S \cdot T=F \cdot \Delta x .
$$

By plugging (21) and (24) into (25), we arrive at

$$
F=G \cdot \frac{M m}{R^{2}}=m \cdot g_{R}
$$

Local gravity is thus a consequence of light-induced quantum-events and the second law.

\subsection{Einstein Equation}

Let a test-system at small distance $R$ be actualized by exchanging photons with $M$ and consequently feel the acceleration $g_{R}$. The energy-emission by the photons must appear in the local rest-frame of the accelerated system as a spontaneous emission from a heat bath in the environment. The temperature is $T_{g_{R}}(20)$, since the period of the corresponding thermal clock must be synchronous with the one of the underlying light-clock (14). This synchronization amounts by (18) to the equation

$$
\frac{4}{h} k_{B} T_{g_{R}} \frac{c}{g_{R}}=\frac{1}{\pi^{2}} .
$$

With $E=M c^{2}, l_{P}=\sqrt{\frac{G \hbar}{c^{3}}}$ and $A_{R}=4 \pi R^{2}$ we derive from (27)

$$
k_{B} T_{g_{R}} A_{R}=4 l_{P}^{2} E \text {. }
$$

By using (20), this leads to

$$
g_{R} A_{R}=\frac{4 \pi G}{c^{2}} E
$$

We are interested in a dynamic development of (29). In the sequel we will continue to work in the local inertial coordinate-chart around the origin $(M)$ and develop Einstein's equation for the $o o(t t)$-component of the metric-tensor. This will suffice to reveal the structure of the equation. With $V_{R}(t)$ denoting the volume of a small ball of test-systems at radius $R(t)$ around the origin, with $R(0)=R, \dot{R}(0)=0$ and $\ddot{R}(0)=g_{R}$, we can rewrite (29) as $[20,21]$

$$
\left.\frac{d^{2}}{d t^{2}}\right|_{t=0} V_{R}=\frac{4 \pi G}{c^{2}} E .
$$


If we introduce the energy-momentum tensor $T_{a b}, 0 \leq a, b \leq 3$, with zero-component $T_{00}=\lim _{R \rightarrow 0} \frac{E_{R}}{V_{R}},{ }^{16}$ denoting the energy density at the origin, and use the local properties of the Ricci tensor $R_{a b}, 0 \leq a, b \leq 3$, we have at the origin [21, 22] (see appendix)

$$
\left.\frac{\ddot{V}_{R}}{V_{R}}\right|_{t=0} \stackrel{R \rightarrow 0}{\rightarrow} c R_{00}^{2}
$$

i.e.

$$
R_{00}=\frac{4 \pi G}{c^{4}} T_{00}
$$

In a transaction there is a transfer of four-momentum through photons connected to a quantum-event. In paragraph 3 we called this momentum-transfer "event-radiation". In order to synchronize local light-clocks (27) we have so far only made use of the energy (zero)-component of event-radiation. Let $A_{i}, i=1 \ldots 3$, be small surface elements with $\left\langle\vec{n}_{A_{i}} \mid e_{j}\right\rangle=0, i \neq j$. From the 3 -momentum there arises pressure in the spatial-directions, which defines the Laue-scalar at the origin

$$
T=\sum_{i=1}^{3} T_{i i}=\lim _{A_{i} \rightarrow 0} \sum_{i=1}^{3} \frac{F_{i}}{A_{i}}=\lim _{A_{i} \rightarrow 0} \sum_{i=1}^{3} \frac{1}{A_{i}} \frac{d p_{i}}{d t} .
$$

This quantity also contributes to the energy density in (32). Let $N_{R}(t)$ be the number of actualizations within volume $V_{R}$ at time $t$. We have with $x_{0}=c t, \tilde{N}_{R}\left(x_{0}\right)=N_{R}\left(\frac{x_{0}}{c}\right)$ and the de Broglie-relation $|\vec{p}|=\frac{h}{R}$

$$
T=-3 \frac{d N_{R}(t)}{d t A_{R}} \cdot \frac{h}{R}=-3 \frac{c \cdot h}{3} \cdot \frac{d \tilde{N}_{R}\left(x_{0}\right)}{d x_{0} V_{R}}==-c \cdot h \cdot \frac{d \lambda\left(x_{0}\right)}{d x_{0}} .
$$

The function $\lambda\left(x_{0}\right)=\frac{\tilde{N}_{R}\left(x_{0}\right)}{V_{R}}$ denotes the number of events per 3 -volume at time $x_{0}$ and therefore $\frac{d \lambda\left(x_{0}\right)}{d x_{0}}$ is the change-rate of actualizations per 3-volume. We assumed in (34) that $\lambda\left(x_{0}\right)$ is constant over 3-space (i.e. in particular independent of $R$ ), which amounts to the homogeneity and isotropy of space with respect to actualizations. We have also tacitly assumed that $\lambda\left(x_{0}\right)$ is a differentiable function in $x_{0}$. This is an assumption, which cannot hold in the quantum-realm, since events represent discrete sets and are not deterministic, but obey a random-process. The only known Lorentz-invariant stochastic law for the spreading of events in $\mathbb{M}^{4}$, such that $N \sim V$, is a Poisson-process with constant average (photon) transaction-rate $\varrho_{\gamma}$ [23]. The homogeneity and isotropy of space-time are thus an

$\overline{16}$ We assume $E$ to be homogeneously distributed over $V_{R}$. 
immediate consequence of this law. Hence, in the above terminology we have for the averages (expectation values) and $\Delta x_{0}>0$

$$
\bar{\lambda}\left(x_{0}+\Delta x_{0}\right)=\bar{\lambda}\left(x_{0}\right)+\varrho_{\gamma} \cdot \Delta x_{0}
$$

So by (35) we can define in analogy to (34)

$$
\bar{T}_{\gamma}=-3 \frac{c \cdot h}{3} \cdot \frac{\Delta \bar{\lambda}\left(x_{0}\right)}{\Delta x_{0}}=-c \cdot h \cdot \varrho_{\gamma} .
$$

If we set $T=\left(T_{00}-\bar{T}_{\gamma}\right)$ we can complete the right hand side of (32) to

$$
\frac{4 \pi G}{c^{4}} T_{00} \rightarrow \frac{8 \pi G}{c^{4}}\left(T_{00}-\frac{1}{2} T \delta_{00}\right) .
$$

We may alternatively shift the added amount $\bar{T}_{\gamma}$ to the left of (32). We have by (36)

$$
\frac{4 \pi G}{c^{4}} \bar{T}_{\gamma}=-\frac{4 \pi G h}{c^{3}} \varrho_{\gamma}=-8 \pi^{2} l_{P}^{2} \varrho_{\gamma}
$$

Therefore, with

$$
\Lambda=8 \pi^{2} l_{P}^{2} \rho_{\gamma}
$$

the synchronization-equation takes the form ${ }^{17}$

$$
R_{00}+\Lambda \delta_{00}=\frac{4 \pi G}{c^{4}} T_{00} .
$$

Note that $\Lambda$ has the dimension of $\frac{1}{(\text { length })^{2}}$. If matter-energy does not only stem from a static mass $M$, but from more complicated material systems, which also exercise pressure $T$, we finally get our main result by repeating the procedure in (37)

$$
R_{00}+\Lambda \delta_{00}=\frac{8 \pi G}{c^{4}}\left(T_{00}-\frac{1}{2} T \delta_{00}\right)
$$

Under the assumption of known transformation rules, the full Einstein equations

$$
R_{\mu \nu}+\Lambda g_{\mu \nu}=\frac{8 \pi G}{c^{4}}\left(T_{\mu \nu}-\frac{1}{2} T g_{\mu \nu}\right)
$$

are equivalent to the fact that (41) holds in every local inertial coordinate system around every point in space-time [22]. Note that from (29) to (41) we followed a path of successive generalization. If we now want to take the reverse way and recover Newtonian gravity from (41), we have to work with masses at low speed

$\overline{17}$ These are the Einstein equations with the metric signature convention $(+---)$. 
$T \ll T_{00}$ and omit $\Lambda$ to get to (32) and then chose weak gravitational fields, leading approximately to Euclidean space and (29). ${ }^{18}$

\section{Summary and Conclusions}

Starting from the idea, that "the potential for a position in space" is a unique identifying property of material systems and that to change values of that property requires the depenence on a logical, a priori system-dependent parameter, we have introduced the pair "space and time" in different models of nature. We have further seen, that the mechanism of electromagnetically induced quantum-events and the corresponding event-radiation unite space and time and do the following: quantum-events (a) actualize matter at space-time points (regions) and (b) induce a universal entropic acceleration on material systems. Event radiation (c) induces naturally intrinsic light-clocks, measuring the rhythm of events and (d) induces a universal repulsive energy density between events. The postulate to synchronize clocks in the accelerated reference frames, ultimately to keep the speed of light constant, then induces a dynamic metric relation on events, governed by Einstein's equation.

Empirical space-time, which we observe, is therefore emerging from a preempirical quantum world, which gets continually actualized by electromagnetic interaction, and which induces by the mechanism of quantum-events a universal entropic force, called gravity, on actualized material systems and a dynamic metric relation between them. Such a universe is a dynamic place, which continually changes in a time-direction, defined by event-radiation, and spatially expands by the momentum exchange in event-radiation. This mechanism explains the close formal relation between gravity and electromagnetism, like the expansion of gravitational influence at the speed of light. It also explains why gravitating and inertial mass are identical, or the fact that the length of a system's world-line measures its physical time [24]. It becomes also clear, that the large scale structure of the universe is a consequence of the long-range nature of the electromagnetic force.

As mentioned in the introduction, there are at present several competing ontological models of space-time, one of which was developed in this paper. It is probably fair to say that at this point in time there is no rigorous proof nor compelling empirical evidence to decide for the correctness of one or the other and the odds are open. There remains, however, the confidence that some day with the progress of theoretical and experimental science we will know the true nature of space-time. This will be a great moment in the history of human thinking about the universe.

\footnotetext{
18 Note that Eq. (32) was Einstein's first proposal for the field equations. It fails because of an incongruancy of the number of degreess of freedom between the components of the metric tensor $g_{\mu \nu}$ and the Ricci tensor $R_{\mu \nu}$.
} 


\section{Appendix}

For the sake of completeness, we want to indicate how to derive the key relation (31). For this we follow the exposition in [21]. Let two nearby particles at relative rest to each other start to fall freely. If the initial velocity of particle one was $v$, then the one of the second particle follows from parallel transport along the connecting vector $\varepsilon u$. If we compare the two velocities after some small time $\varepsilon$, then the first one moved along $\varepsilon v$ and we have to again parallel transport it to $v_{1}$ in order to compare it to the corresponding $v_{2}$. Over the passage of time the average relative acceleration of the two particles $a_{\varepsilon}$ is $a_{\varepsilon}=\frac{v_{2}-v_{1}}{\varepsilon}$. By the definition of the curvature tensor $R$ there holds

$$
\lim _{\varepsilon \rightarrow 0} \frac{v_{2}-v_{1}}{\varepsilon^{2}}=R(u, v) v .
$$

Hence, by the symmetries of the tensor $R$,

$$
\lim _{\varepsilon \rightarrow 0} \frac{a_{\varepsilon}}{\varepsilon}=-R(v, u) v
$$

or in coordinate-components

$$
\lim _{\varepsilon \rightarrow 0} \frac{a_{\varepsilon}^{j}}{\varepsilon}=-R_{k l m}^{j} v^{k} u^{l} v^{m} .
$$

A small ball $V_{R}$ of test particles, starting at relative rest and moving geodesically, changes in second order to an ellipsoid whose axes initially don't rotate. We can therefore choose local inertial coordinates in which (to second order) the center of the ball doesn't move and the principal axes of the ellipsoid stay aligned with the coordinate axes. If the ball's initial radius is $\varepsilon$, then $r^{j}(t)=\varepsilon+\frac{1}{2} a^{j} t^{2}+O\left(t^{3}\right)$. Hence

$$
\lim _{t \rightarrow 0} \frac{\ddot{r}^{j}}{r^{j}}=\frac{a_{\varepsilon}^{j}}{\varepsilon} .
$$

With $u$ denoting the unit-vector in $j$-direction and $v$ the one in time-direction we have by $\mathrm{C} 3$ without summation over $j$

$$
\operatorname{limlim}_{\varepsilon \rightarrow 0 t \rightarrow 0} \frac{\ddot{r}^{j}(t)}{r^{j}(t)}=-R_{t j t}^{j} .
$$

Since the volume of our ball is proportionate to the radii, $\lim _{t \rightarrow 0}\left(\left.\frac{\ddot{V}}{V}\right|_{t}\right)=\lim _{t \rightarrow 0} \sum_{j} \frac{\ddot{r j}^{j}(t)}{r^{j}(t)}$, so with summation over all four $j$ (since $\left.R_{t t t}^{t}=0\right)^{19}$

$$
\left.\lim _{V \rightarrow 0} \frac{\ddot{V}}{V}\right|_{t=0}=-R_{t j t}^{j}=R_{00} .
$$

$\overline{19 \text { We choose the convention } R_{\mu \nu}}=R_{\mu \nu \lambda}^{\lambda}=-R_{\mu \lambda \nu}^{\lambda}$. 
The factor $c^{2}$ in front of the Ricci-curvature stems from the line-element $d \tau=c d t$.

Open Access This article is licensed under a Creative Commons Attribution 4.0 International License, which permits use, sharing, adaptation, distribution and reproduction in any medium or format, as long as you give appropriate credit to the original author(s) and the source, provide a link to the Creative Commons licence, and indicate if changes were made. The images or other third party material in this article are included in the article's Creative Commons licence, unless indicated otherwise in a credit line to the material. If material is not included in the article's Creative Commons licence and your intended use is not permitted by statutory regulation or exceeds the permitted use, you will need to obtain permission directly from the copyright holder. To view a copy of this licence, visit http://creativecommons.org/licen ses/by/4.0/.

\section{References}

1. Barbour, J.: The End of Time, 1st edn. Oxford University Press, Oxford (1999)

2. Grünbaum, A.: Philosophical Problems of Space and Time, 2nd edn. Boston Studies in the Philosophy and History of Science, Boston (1973)

3. Kastner, R.E.: The possibilist transactional interpretation and relativity. Found. Phys. 42(8), 10941113 (2012)

4. Sorkin, R.D.: Geometry from order: causal sets. Einstein Online 02, 1007 (2006)

5. Knuth, K., Bahreyni, N.: A potential foundation for emergent space-time. J. Math. Phys. 55, 112501 (2014)

6. Blanchard, Ph., Fröhlich, J., Schubnel, B.: A 'Garden of Forking Paths': the quantum mechanics of histories of events. Nucl. Phys. B 912, 463-484 (2016)

7. Kastner, R.: Unitary-only quantum theory cannot consistently describe the use of itself: On the Frauchiger-Renner paradox. Found. Phys. 50, 441 (2019)

8. Cramer, J.G.: The transactional interpretation of quantum mechanics. Rev. Mod. Phys. 58(3), 647687 (1986)

9. Kastner, R.E.: Cramer's transactional interpretation ans causal loop problems. Synthese 150(1), 1-14 (2006)

10. Kastner, R.E., Cramer, J.G.: Quantifying absorption in the transactional interpretation. Int. J. Quantum Found. 4(3), 210-222 (2018)

11. Margolus, N., Levitin, L.B.: The maximum speed of dynamical evolution. Physica D 120, 188-195 (1998)

12. Zinkernagel, H.: Did time have a beginning? Int. Stud. Philos. Sci. 22(3), 237-258 (2008)

13. Rovelli, C.: "Incerto tempore incertisque loci": can we compute the exact time at which a quantum measurement happens? Found. Phys. 28(7), 1031-1043 (1998)

14. Kastner, R.E.: The Transactional Interpretation of Quantum Mechanics. Cambridge University Press, Cambridge (2013)

15. Rovelli, C., Smerlak, M.: Thermal time and the Tolman-Ehrenfest effect: temperature as the speed of time. Class. Quantum Gravity 20(7), 075007 (2011)

16. Unruh, W.G.: Notes on black-hole evaporation. Phys. Rev. D 14, 870 (1976)

17. Davies, P.C.W.: Scalar particle production in Schwarzschild and Rindler metrics. J. Phys. A Math. Gen. 8, 609-616 (1975)

18. Verlinde, E.: On the origin of gravity and the laws of Newton. JHEP 4, 29 (2011)

19. Bekenstein, J.D.: Black holes and entropy. Phys. Rev. D 7, 2333 (1973)

20. Susskind, L.: The world as a hologram. J. Math. Phys. 36(11), 6377-6396 (1995)

21. Fischer, K.: Einsteins-Gleichung der Gravitation ausgedrückt in Tensoren. In: Fischer, K. (ed.) Relativitätstheorie in einfachen Worten, pp. 160-161. Springer Spektrum, Berlin (2009)

22. Baez, J., Bunn, E.: The meaning of Einstein's equation. Am. J. Phys. 73(7), 644-652 (2007)

23. Bombelli, L., Henson, J., Sorkin, R.D.: Discreteness without symmetry breaking: a theorem. Mod. Phys. Lett. A 24(32), 2579-2587 (2006) 
24. Fletcher, S.C.: Light clocks and the clock hypothesis. Found. Phys. 43(11), 1369-1383 (2013)

Publisher's Note Springer Nature remains neutral with regard to jurisdictional claims in published maps and institutional affiliations. 